\title{
A Distributed GPU-Based Framework for Real-Time 3D Volume Rendering of Large Astronomical Data Cubes
}

\author{
A. H. Hassan ${ }^{\mathrm{A}, \mathrm{C}}$, C. J. Fluke , and D. G. Barnes $^{\mathrm{B}}$

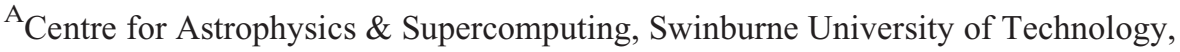 \\ Hawthorn, Vic. 3122, Australia \\ ${ }^{\mathrm{B}}$ Monash e-Research Centre, Monash University, Clayton, Vic. 3800, Australia \\ ${ }^{\mathrm{C}}$ Corresponding author. Email: ahassan@swin.edu.au
}

\begin{abstract}
We present a framework to volume-render three-dimensional data cubes interactively using distributed ray-casting and volume-bricking over a cluster of workstations powered by one or more graphics processing units (GPUs) and a multi-core central processing unit (CPU). The main design target for this framework is to provide an in-core visualization solution able to provide three-dimensional interactive views of terabyte-sized data cubes. We tested the presented framework using a computing cluster comprising 64 nodes with a total of 128 GPUs. The framework proved to be scalable to render a $204 \mathrm{~GB}$ data cube with an average of 30 frames per second. Our performance analyses also compare the use of NVIDIA Tesla 1060 and $2050 \mathrm{GPU}$ architectures and the effect of increasing the visualization output resolution on the rendering performance. Although our initial focus, as shown in the examples presented in this work, is volume rendering of spectral data cubes from radio astronomy, we contend that our approach has applicability to other disciplines where close to real-time volume rendering of terabyte-order three-dimensional data sets is a requirement.
\end{abstract}

Keywords: methods: data analysis — techniques: miscellaneous

Received 2012 March 13, accepted 2012 May 1, published online 2012 May 22

\section{Introduction}

Radio astronomy is entering a new data-rich era. Upcoming facilities such as the Australian Square Kilometre Array Pathfinder (ASKAP: Johnston et al. 2008), MeerKAT (Booth et al. 2009), the Low Frequency Array (LOFAR: Röttgering 2003), and ultimately the Square Kilometre Array $(\mathrm{SKA})^{1}$ will enable astronomers to observe the radio universe at an unprecedented spatial and frequency resolution. Unfortunately, handling the data from these facilities, expected to be of terabyte order for individual observations, will pose a significant challenge for current astronomical data analysis and visualization tools (e.g. DS9 ${ }^{2}$ and $\mathrm{CASA}^{3}$ ). Such data volumes are orders of magnitude larger than astronomers and existing astronomy software are accustomed to dealing with.

Perhaps as never before, opportunities exist for approaches based on advances in computing hardware and a wider adoption of techniques centred on computer science and scientific computing to overcome these challenges. Of particular interest is the availability of graphics processing units (GPUs) as low-cost, highly parallel, streaming co-processors. These allow implementation of

\footnotetext{
1 http: / /www.skatelescope.org/

${ }^{2}$ http : / / hea-www . harvard. edu/RD/ds9 /

${ }^{3}$ http: / / casa.nrao.edu/
}

specific algorithms for visualization and analysis that may not have been (computationally) practical for CPU-only distributed systems.

\subsection{Spectral Data Cube Visualization}

Today, most radio astronomers still rely on two-dimensional (2D) techniques to visualize spectral data cubes that are inherently three-dimensional (3D): two spatial axes and one frequency or velocity axis. These $2 \mathrm{D}$ techniques display data cubes as a sequence of colour-coded (or flooded-contoured) frames at a reasonable speed to enable astronomers to identify the relationships between these frames, and detect the main features of signal and noise therein. These techniques, which usually rely on holding an entire data cube in CPU memory, are not feasible with the upcoming dataset sizes.

Wider adoption of $3 \mathrm{D}$ visualization techniques has been proposed as an alternative to $2 \mathrm{D}$ techniques, particularly for obtaining global views. Spectral data cubes are often characterised by a lack of well-defined surfaces and the presence of significant features (i.e. sources) with either a spatial or spectral extent near or below the noise level. These data characteristics limit the usage of surface-rendering techniques [e.g iso-surfaces: Beeson, Barnes, and Bourke (2003); Hassan, Fluke, and Barnes 
(2011)], the adoption of multi-resolution algorithms, or general purpose data visualization tools. For a detailed review of the previous work in spectral data cube visualization, we refer the reader to Hassan and Fluke (2010).

In terms of providing global views of data, volume rendering is the most promising candidate, particularly in cases where clear feature segmentation cannot be done (Beeson et al. 2003; Gooch 1995; Oosterloo 1995). Volume rendering is the process of generating a colour-coded $2 \mathrm{D}$ projection of the $3 \mathrm{D}$ data on a user-controlled viewport.

Existing astronomy volume-rendering implementations have addressed the special characteristics of astronomical data, including the selection of transfer functions (Gooch 1995; Oosterloo 1996), effective handling of adaptive grids and different data resolutions (Kaehler et al. 2006; Nadeau et al. 2001; Magnor et al. 2005), and addressing the large data size problem (Beeson et al. 2003). Some of these implementations were directed toward rendering a specific dataset (Kaehler et al. 2006; Nadeau et al. 2001; Magnor et al. 2005), while others demonstrated the usage of their tool with different datasets, or provide their tool to the public domain. Throughout these papers we can find two main implementation trends. The first trend customizes an existing code or uses it as a base for the implementation (Becciani et al. 2000, 2001; Gheller et al. 2002; Becciani et al. 2003; Payne, Jedrzejewski, and Hook 2003; Comparato et al. 2007; Beeson et al. 2003), while the second trend consists of building one's own custom implementation (Gooch 1995; Oosterloo 1996; Nadeau et al. 2001; Magnor et al. 2005).

In most of these cases, the emphasis was on volume rendering a dataset that could fit entirely in the (local) CPU memory - appropriate when considering data sizes measured in tens of megabytes (MB) to a few gigabytes (GB). Handling data cubes larger than a single machine's memory limit was first addressed in astronomy by Beeson et al. (2003), using the distributed shear-warp algorithm (Lacroute and Levoy 1994). Visualization requirements from other disciplines (e.g. real-time interactive response) have motivated progress in parallel and distributed volume-rendering solutions for increasingly larger data sizes.

\subsection{GPU Cluster Volume Rendering}

In general, volume-rendering techniques can be classified based on the order of data traversal into image-order (e.g. ray-casting: Levoy 1988), object-order (e.g. splatting: Westover 1990), and hybrid (e.g. shear-warp factorization: Lacroute and Levoy 1994). For details on each of these categories and different volume-rendering parallel and distributed implementation trials, we refer the reader to Schwarz (2007); Molnar et al. (2008).

The ray-casting process aims to map each pixel on the viewing plane to a colour. The colour and the opacity of each volume element (voxel) are derived from its data value using a predefined mapping operator called a 'transfer function'. For each pixel on the viewing plane, the ray-casting process computes a ray originating at this pixel and shoots it into the data volume. By tracing this ray and accumulating the colour and opacity values along the ray, the ray-casting process computes and assigns a final colour and opacity to the pixel. See Levoy (1990) for a detailed description of the original ray-casting algorithm.

Although it is a computationally intensive task, raycasting has a simple and clear parallel nature. This parallel nature has motivated the development of number of parallel ray-casting algorithms with special attention to the usage of GPUs (e.g. Goel and Mukherjee 1996; Scharsach 2005; Strengert et al. 2006; Maximo et al. 2008; Humphreys et al. 2008; Eilemann, Makhinya, and Pajarola 2008; Jin et al. 2010). An extended survey of research on high-performance volume rendering using raycasting and the other alternative rendering approaches can be found in Marmitt, Friedrich, and Slusallek (2008).

Using a GPU cluster to perform volume rendering using ray-casting performed in the fragment shader unit and volume-bricking was addressed by Muller, Strengert, and Ertl (2006, 2007). They investigated the effect of using empty-space skipping, static and dynamic loadbalancing approaches, and uniform and non-uniform bricking on the frame rendering time. Stuart et al. (2010) discussed the usage of the MapReduce workflow to implement multi-GPU volume rendering. Their implementation provides both in-core and out-of-core volume rendering based on a $\mathrm{CUDA}^{4}$ implementation of the ray-casting algorithm.

Many of the previously mentioned trials use OpenGL ${ }^{5}$ to implement different rendering tasks (e.g. depth sorting) and the implementation of the volume-rendering part. That allows them, indirectly, to use the distributed processing capabilities of GPUs. Within this work, we utilized CUDA to develop our volume-rendering algorithm and all the associated rendering tasks. While this made the development task harder, the selection of CUDA gives us two main advantages over OpenGL-based distributed rendering. It enables us, relatively more easily, to develop other data analysis and processing tasks that utilize the data already in the GPU memory and the GPU's processing power (e.g. calculating data minimum and maximum values). Also, using CUDA enables our framework to work without the need for XWindows ${ }^{6}$ or OpenGL, which are not supported by some high-performance GPUs or due to operational restriction are not easily supported over non-visualization-oriented supercomputers (e.g. the previous NVIDIA Tesla Family S1060 and S1070). We think the ability to use general purpose GPU-based supercomputers and clusters (e.g. the CSIRO GPU Cluster ${ }^{7}$ and the gStar Supercomputer ${ }^{8}$ ) is an important feature to enable

\footnotetext{
${ }^{4}$ http: / / www.nvidia.com/cuda

5 http: / / www . opengl.org/

${ }^{6}$ http: / / www.x.org

${ }^{7}$ http: / / WwW.csiro.au/resources/GPU-cluster.html

8 http: / / astronomy.swin.edu.au/supercomputing / green $2 /$
} 
more astronomers to utilize $3 \mathrm{D}$ visualization in their dayto-day data analysis and quality control tasks.

\subsection{An Improved Solution}

We present an in-core solution for interactive volumerendering datasets that exceed the single-machine memory limit by using a distributed GPU infrastructure and the ray-casting technique. This work represents both enhancement and an extension to our previously published work (Hassan et al. 2011). While the hardware architecture remains as in figure 2 of Hassan et al. (2011), we have introduced some significant changes to the software architecture to reduce the communication overhead and to speed up the total rendering time. The main contributions of this work are as follows.

- Utilize GPU texture memory to speed up the raycasting process and to facilitate the usage of trilinear interpolation.

- Investigate the framework's scalability up to 128 GPUs and its ability to render up to $200 \mathrm{~GB}$ data cubes.

- Change the data partitioning to utilize $k$-dimensional trees ( $k$-d trees) to enable fair data distribution over the contributing nodes.

- Utilize the rendering rectangle concept further to minimize the communication overhead between different nodes and the server.

- Utilize asynchronous communication to overlap communication with the merging process.

- Implement the server merging process over GPU to speed it up and minimize the merging overhead.

- Dynamic selection of the sampling step to speed up the ray-casting process while maintaining the rendering accuracy.

Overall, these changes enhance the system's scalability, allowing us to make use of new GPU-based supercomputing clusters and thus interactively visualize even larger data sizes (200 GB compared with $25 \mathrm{~GB}$ with our previous solution) and reduce the total rendering time, enabling us to achieve frame rates around 30 frames per second (fps) compared with 5 fps with our previous solution.

\section{Distributed GPU Ray-Casting Framework}

In this section, we present the framework's main software components and related design decisions. Also, we elaborate on the new features presented in this work.

\subsection{Design Philosophy}

The GPU execution model follows a master-slave like execution paradigm. The CPU acts as the main execution controller, controls the access to main memory, pushes the data to the GPU local memory, invokes GPU execution, and pulls the results back to the main memory. On the other hand, a GPU is an order of magnitude faster than the CPU in executing single program multiple data (SPMD) kinds of operation. The lack of direct access to the main memory, the limited communication bandwidth between CPU and GPU, and the limited local GPU memory size (currently $6 \mathrm{~GB}^{9}$ at maximum) are the main factors restricting the use of GPUs in real-time processing/visualization of larger-than-memory datasets. To address some of these issues, the latest generation of GPUs (e.g. NVIDIA Fermi model) are able to communicate and exchange data between each other with limited CPU involvement. ${ }^{10}$ Although such improvements can be effective with GPUs that share the same memory address space (on average 2 GPUs per node), this cannot be easily extended to address the communication between GPUs within different nodes.

Managing each GPU as a separate instance that synchronizes and exchanges data using the message-passing interface (MPI) ${ }^{11}$ standard is a straightforward technique to target such architectures. While the message-passing mechanism is generic enough to deal with such situations, the communication overhead caused by the MPI implementation will reduce the system performance. This overhead might not be noticeable while using CPUs, but it is relatively high, especially when tens of synchronization and data exchange messages are required per second.

One of the main design objectives of the framework presented is to offer two modes of communication: an asynchronous shared-memory type of communication between GPUs in the same memory-address space and a message-passing type of communication between GPUs connected via a network. This combination of shared memory communication and distributed memory communication, and the overlapping between computation and communication, reduces the communication overhead and reduces the needed execution time.

The process of result display is performed outside the backend compute cluster for a practical reason. Usually, large GPU cluster (or high-performance computing) facilities do not include appropriate display facilities that can support a high-level output resolution or interactivity for the user. Furthermore, some of the high-end highperformance computing GPUs do not contain appropriate interfaces for display devices (e.g. NVIDIA Tesla 1060 or 1070 cards).

To achieve the required volume-rendering output, each GPU thread is executing the ray-casting algorithm to map a single pixel on the output image into a colour value. The selection of ray-casting was based upon the following algorithmic advantages.

1. The ray-casting algorithm is primarily an image-order volume-rendering algorithm, which means its complexity mainly depends on the output image size rather than the input data size. The data size resolution does play a part in determining the number of samples

\footnotetext{
${ }^{9}$ http: //www.nvidia.com/object/preconfiguredclusters.html

${ }^{10}$ http: // developer. download.nvidia.com/compute/ cuda/4_0/CUDA_Toolkit_4.0_Overview.pdf

${ }^{11}$ http: / / www . mpi-forum. org/docs /mpi-20-html/mpi2report.html
} 
Server Node

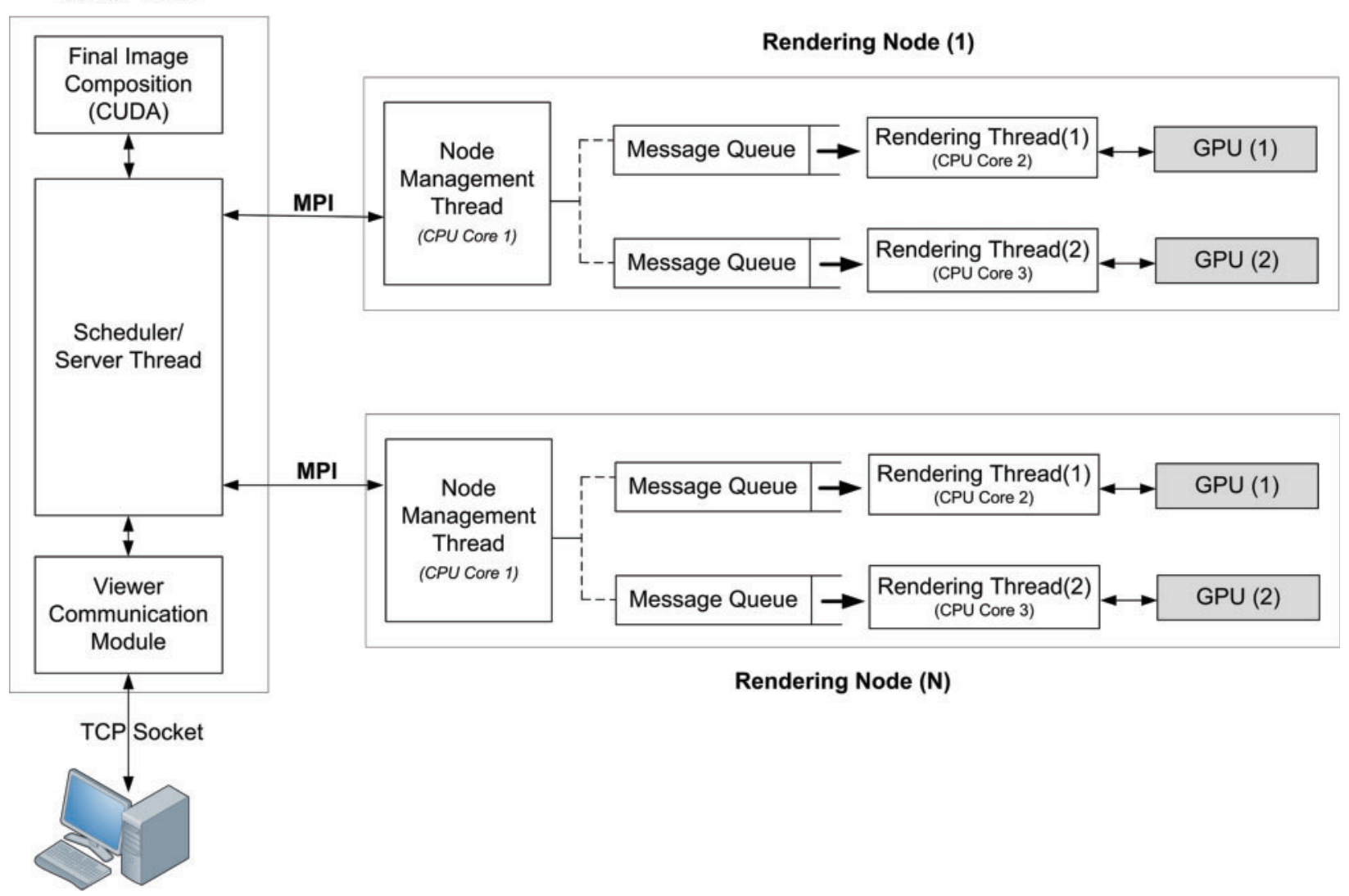

Figure 1 Schematic diagram for the main software components of the proposed framework. The communication between the viewer application and the server is done using TCP sockets and is initiated by the viewer application. The communication between the server node and the rendering node is done using MPI. Each GPU is managed and controlled by a separate CPU thread. The internal communication between different GPUs threads within the same workstation is done using a custom message queue and is managed by the root thread.

needed per ray, but this has a minor impact, as is shown in Section 3.

2. The ray-casting algorithm is an embarrassingly parallel algorithm, and is well-matched to the GPU processing paradigm.

On the other hand, the disadvantages of using an image-order volume-rendering methodology are as follows.

1. An image-order volume-rendering algorithm requires the entire dataset to be accessible during the rendering process. This was solved using the bricking technique and proxy geometry (see Lombeyda et al. 2001 for more details).

2. The bricking technique independently maps each subvolume into the full resolution output frame. Hence, each GPU is required to evaluate the same number of output pixel values. While the proxy geometry is usually used to early-terminate those rays falling outside the current volume, this kind of conditional execution is poorly supported by the GPU architecture and reduces the overall performance. ${ }^{12}$ This was

\footnotetext{
${ }^{12}$ http: / / developer.download.nvidia.com/compute/ cuda/4_0_rc2/toolkit/docs/CUDA_C_Programming_ Guide.pdf-chapter 4.
}

addressed by using a CPU-level global termination criterion (rendering rectangle), which minimizes the need for early ray termination on the GPU level.

3. The main bottleneck for distributed image-ordered volume-rendering methodologies is the compositing operation. We solved this issue by partitioning the compositing into two-stage compositing, which reduces the communication overhead and the number of tasks that need to be performed by the server node. Also, we utilized rendering rectangles to minimize the number of compositing operations required, and moved the composition step from CPU to GPU.

\subsection{Hardware and Software Architecture}

The main software components of the system are shown in Figure 1. These components are as follows.

1. Viewer communication module. This module is based on TCP sockets to enable the server to exchange messages with the viewer machine(s). Messages are exchanged in a custom, predefined binary format. The main task for this module is to interpret the message, identify the required parameters, and notify the server using an event-driven architecture. This module implements a state machine to identify and validate the possible client communication scenarios. 
2. Server scheduler module and main thread. This module is the main system backbone. It is responsible for partitioning the data cube into sub-cubes, assigning different sub-cubes to rendering nodes and GPUs, synchronizing between different rendering nodes, utilizing the global image-composition module to generate the final rendering output, and communicating with the viewer application using the viewer communication module. See Figure 2 for details of the different tasks.

3. Global image-composition module. This module is mainly a CUDA driver API wrapper that contains the main functionality required to composite different output sub-frames (received from rendering clients).

4. Rendering node - management thread. This thread is responsible for initiating and monitoring different

\section{Distributed Volume Rendering Framework}

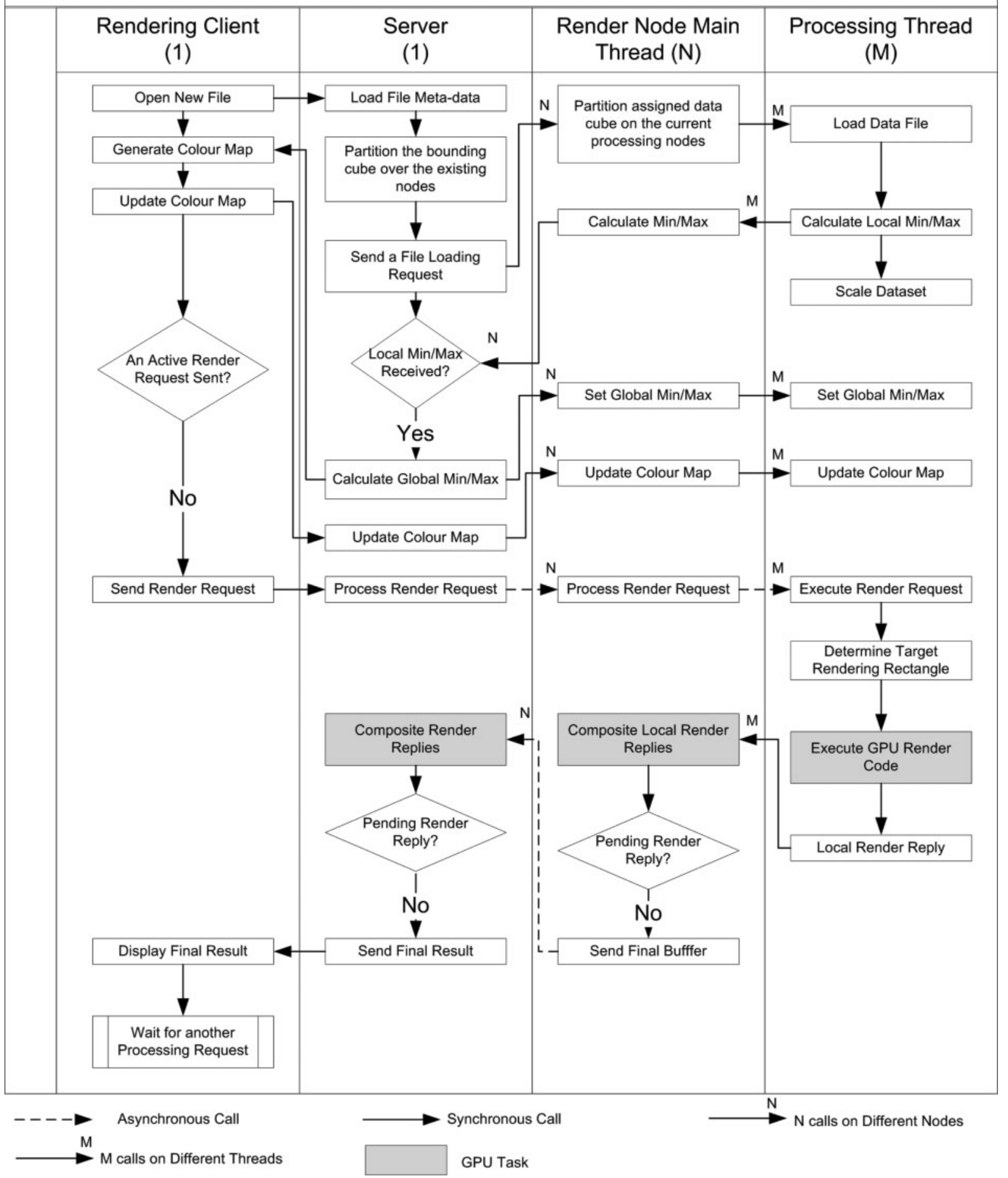

Figure 2 Cross-functional diagram showing the interactions between the system's software components. 
rendering threads (one for each GPU in its node), controlling MPI messages and broadcasting them to the rendering threads, compositing the output of the rendering threads, and sending the rendering results back to the server.

5. Rendering node - rendering thread(s). Each of these threads is responsible for managing one GPU card, using the CUDA driver API. These threads are responsible for loading the data and transferring it to GPU texture memory, determining the associated rendering rectangle for each rendering request, and clipping the ray-casting process to it, performing the actual rendering via the GPU, and receiving the output frame back for compositing.

Further details about these modules, and how they communicate, is shown in Figure 2 and is discussed in detail in the next section. For a detailed description of the hardware architecture, we refer the reader to Section 2 and figure 2 of Hassan et al. (2011).

\subsection{Main Framework Processes}

We now describe the framework's main processes and provide a highlight of the details of each software components and how they are integrated together within the framework.

\subsubsection{Data Partitioning and Scheduling}

The input data are partitioned into two levels (see Figure 3). The first level partitions the input data cube into smaller sub-cubes based on the number of processing nodes. The size of these sub-cubes should fit in each node's memory, and the total available GPU memory on each node. The second level further partitions each node sub-cube into smaller sub-cubes, which are mapped to the GPUs of each node. The problem is further partitioned by mapping each pixel of the output frame to a GPU thread.

The input-data partitioning process is performed over the server using the file metadata. Based on the file metadata, the input cube is partitioned into a set of uniform sub-cubes using a k-d tree partitioning. The main target of this step is to partition the global data cube into equal volume partitions, which distributes the required computations fairly over the contributing GPUs. In the case in which the current number of available GPUs does not match the number of nodes required for a balanced k-d tree, the data are partitioned into two or more balanced $\mathrm{k}$ - $\mathrm{d}$ trees, where the longest axis is used to partition the
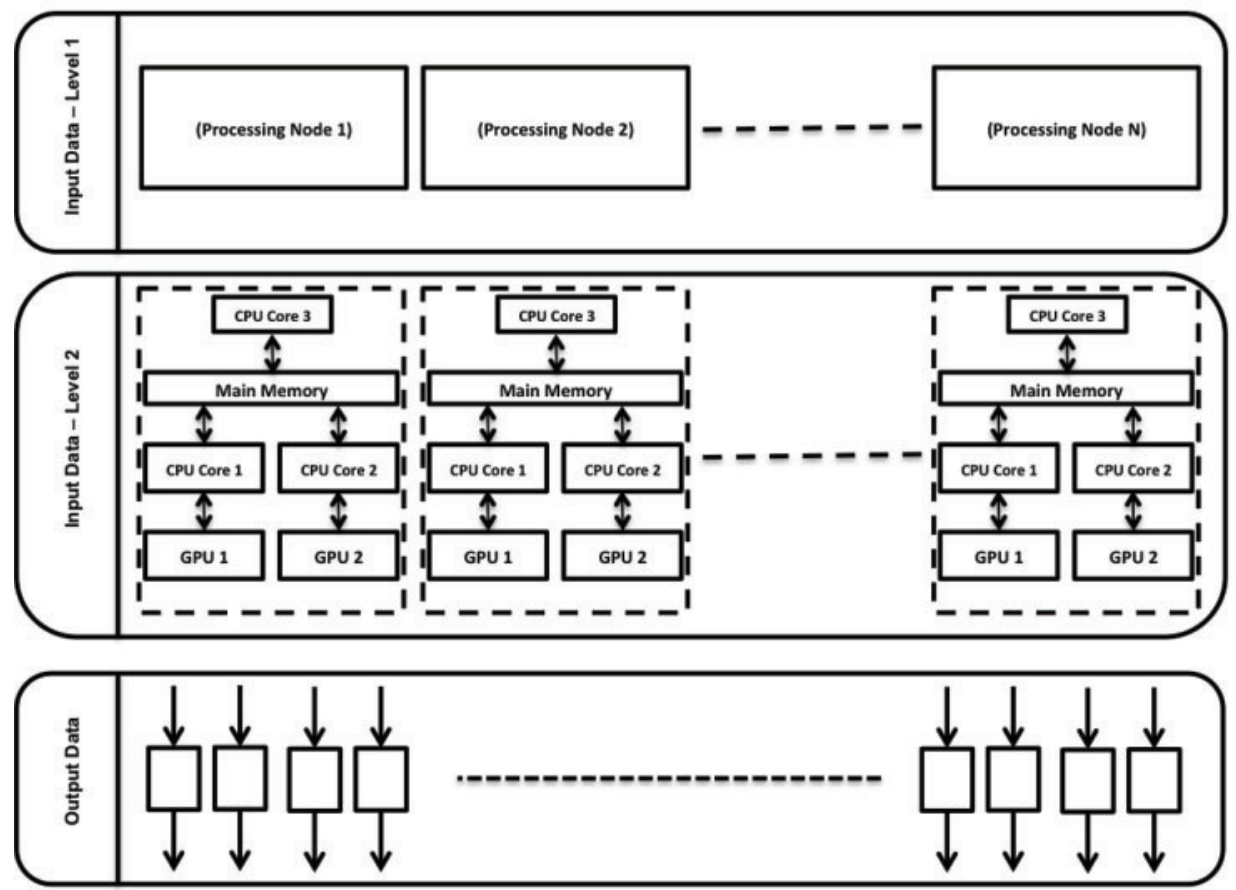

Figure 3 Illustration of the data partitioning using the k-d tree subdivision and different levels of granularity. The first level of granularity is the node level (4 nodes in the example). The second level further partitions each node's sub-cube into smaller sub-cubes, which are mapped to the GPUs ( 8 GPUs with 2 Nodes per GPU). On the GPU level the resultant image is partitioned, where each pixel is allocated to a GPU thread to process. 


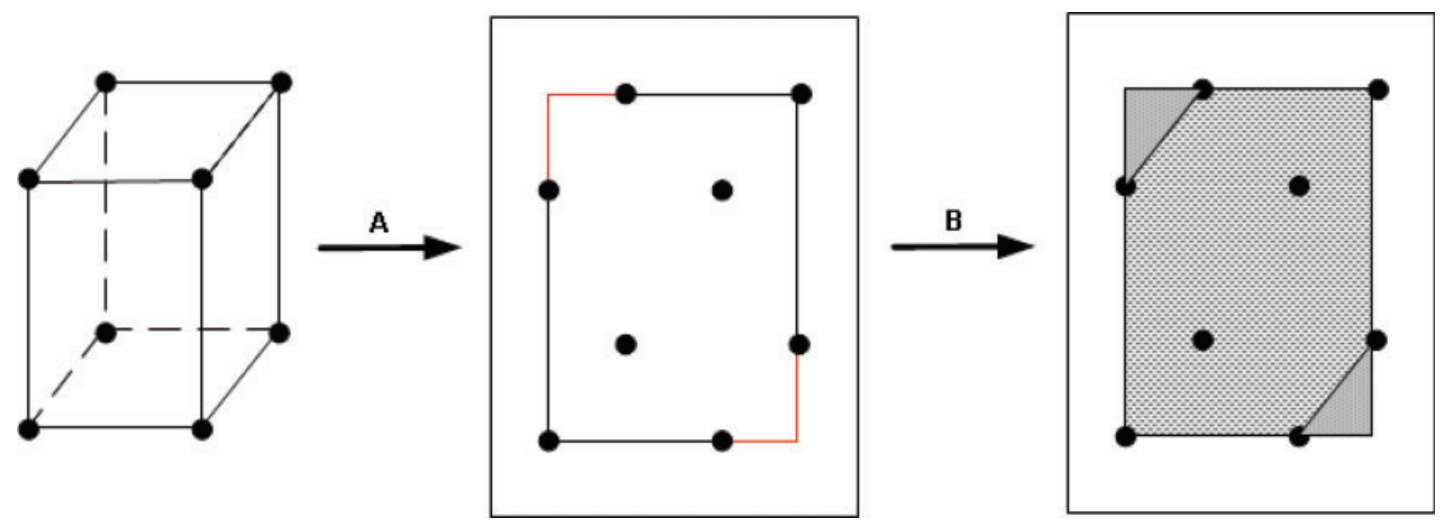

Figure 4 Illustration of the process of determining the rendering rectangle for each GPU. (a) A 2D projection of the cube points is calculated, followed by calculating the bounding rectangle. (b) The bounding-box check is used to exclude the gray region from the output.

main cube into the root of each of these k-d trees. The partition point is selected based on the ratio of the number of GPUs assigned to each k-d tree to the total number of GPUs available. The previous partition schema discussed in Hassan et al. (2011) was not generic enough to achieve a balanced data partitioning over a large number of nodes and GPUs, which is better handled using k-d trees. This step aims to minimize the differences between sub-cube dimensions (this contributes to the difference in the frame-rendering time with different cube orientation angles, see Section 3 for details) and to achieve a balanced data assignment between different GPUs.

Each rendering node sends an independent file-loading request(s) to the file server and starts the file loading process. Whenever the data are loaded in the nodes' CPU memory, the data transformation starts from the CPU memory to the GPU texture memory. An independent task to determine the local minimum and maximum starts when the data are loaded into the GPU memory, and the result is combined with the 'rendering ready' signal. The output data mapping is updated for each frame based on the proxy-geometry orientation and the computed rendering rectangle.

\subsubsection{Ray-Casting Process}

A significant portion of the ray-casting process is executed on GPU using the NVIDIA CUDA driver API. The lack of predictable data-access behaviour limits the optimized usage of the global GPU memory and dramatically reduces the data-access speed. We choose to store the data cube in the GPU texture memory, which provides the highest available data-access speed for such amounts of data. Also, texture memory provides built-in fast linear interpolation functionality. The ray-casting process starts on the CPU side with limited pre-processing steps, aiming to speed up and optimize the ray-casting process. Using information provided by the viewer application and passed through the server about the size of the final output frame and the OpenGL projection and transformation matrices, each rendering thread projects its data sub-cube vertices onto the output frame. These projected vertices are used as an input to calculate a bounding rectangle, which contains all the points, to limit the ray-casting process to a specific region of the output frame. We call this region the 'rendering rectangle': see Figure 4 for an illustration of the process. The rendering parameters are then passed to the GPU kernel, where a GPU kernel instance is invoked for each pixel within the rendering rectangle.

Each GPU kernel then starts by calculating its ray's start location, orientation, and where it intersects with the bounding cube. The ray entry and exit point is used to determine a suitable sample step size using a discrete raycasting with at least one sample for each voxel intersected [see Kaufman (1998) for details]. This provides acceptable output accuracy and processing time, especially with the lack of huge variation in the data-point values. Also, this check is used to terminate early any ray lying outside the bounding cube (a few rays may pass the rendering rectangle test but still do not intersect with the bounding cube). The ray-casting process proceeds while the exit point is reached, or the maximum pixel value (defined by the global maximum of the data) or opacity is achieved.

\subsubsection{Final Image Composition}

The image-compositing process is performed over two layers. The first layer is on each of the processing nodes that contains more than one GPU. Using a shared rendering buffer, initially blank, each GPU projects its rendering data over this buffer with the guidance of its rendering rectangle. The compositing process is achieved using a GPU kernel, and the rendering rectangles are merged together to form the whole-node rendering rectangle. The whole-node rendering rectangle (a union of the rendering rectangles of each GPU) is then used to clip the rendering buffer, and only the area within the rendering rectangle is sent to the server along with the rendering rectangle information.

The server receives the individual rendering subbuffers into a separate queue to achieve the best overlap between the communication and the process of server merging. Using each node-rendering rectangle, the server 
Table 1. Sample datasets used to evalute the performance of our framework

\begin{tabular}{|c|c|c|c|}
\hline Dataset name & Dimensions (data points) & Source/Credits & File size \\
\hline Nbody cube & $1024 \times 1024 \times 1024$ & $\begin{array}{l}\text { High resolution } 1080^{3} \text { dark matter simulation of a } \\
125 \mathrm{Mpc}^{-1} \text { box by Swinburne Computations for } \\
\text { WiggleZ (SCWiggleZ) project (Poole et al., in } \\
\text { preparation) }\end{array}$ & 4 Gigabyte \\
\hline HIPASS cube & $1721 \times 1721 \times 1025$ & $\begin{array}{l}\text { HIPASS Southern Sky, data courtesy Russell Jurek } \\
\text { HIPASS team }\end{array}$ & 12 Gigabyte \\
\hline GASS cube & $2502 \times 2501 \times 1093$ & $\begin{array}{l}\text { The Parkes Galactic All-Sky Survey, data courtesy } \\
\text { Naomi McClure-Griffiths/ GASS team (McClure- } \\
\text { Griffiths et al. 2009) }\end{array}$ & 25 Gigabyte \\
\hline Scaled Nbody cube & $2600 \times 2600 \times 2600$ & Scaled version of the Nbody cube & 65.4 Gigabyte \\
\hline Scaled GASS cube & $5004 \times 5002 \times 2186$ & Scaled version of the GASS cube & 203.8 Gigabyte \\
\hline
\end{tabular}

merges the node results with the final rendering buffer. The server merging process is also performed over GPU. When all the received sub-buffers are merged, the server initiates a colour-mapping process using the user-specified colour map and sends the results back to the client.

The composition complexity and validity depends on the selected transfer function. The currently adopted transfer function is the maximum intensity projection (MIP: Wallis et al. 1989). It was selected because of its straightforward, easy to interpret, mapping between the data and the colour map used, ability to emphasize features with a local maximum (e.g. large-scale noise patterns and radio frequency interference), and low parallelization overhead. The usage of MIP as the main transfer function allows arbitrary compositing order, which facilitates and speeds up the compositing process. Lombeyda et al. (2001) provide a mathematical proof that the general alpha-blending volume-rendering operator is associative, which allows performing the compositing step in parallel.

\subsubsection{Communication}

The communication overhead is the main bottleneck for the whole system's scalability. After trying different methodologies, including multi-layer compositing (see Beeson et al. 2003), using different dedicated compositing nodes, and even applying different compression algorithms, the direct-send methodology was found to be the best from the perspective of the total rendering time and the cost of the hardware needed. The same conclusion was reached by Stuart et al. (2010), Muller et al. (2006), and Muller et al. (2007).

The average rendering result message size, without compression or encoding, is $4 \mathrm{MB}$ for a $1024 \times 1024$ output frame. This amount of data increases linearly with the number of nodes in this case, which means that adding new GPUs does not necessarily decrease the total frame rendering. Here, using the individual node render rectangle to determine the amount of non-blank data, the amount of data transferred between the nodes and the server is no longer constant.

Based on the rendering rectangles of individual nodes, each node-rendering buffer is packed in a smaller message, the size of which depends on the cube orientation and the amount of data assigned to each node. Furthermore, each node utilizes its local memory to perform the first local composition step which redfurther reduces the amount of data to be transferred to the server. With the message-packing mechanism and the two-stage compositing, the total amount of data transferred for each frame is reduced from $N \times M$ to $M+\varepsilon$, where $N$ is the number of rendering nodes, $M$ is the final frame size in bytes, and $\varepsilon$ is a slight increase in the size caused by the overlapping between different rendering rectangles. This reduction removes a potential bottleneck for the framework scalability and significantly reduces the total rendering time. Also, the usage of rendering rectangles speeds up the server compositing process and allows better overlap between communication and computations because it excludes applying the compositing operator over non-overlapped pixels.

\section{Results and Discussion}

\subsection{Performance Analysis and Timing Tests}

Performance analysis and timing tests were performed on the Australian Commonwealth Scientific and Industrial Research Organisation (CSIRO) GPU cluster. This GPU cluster contains 128 nodes with two GPUs each. The nodes are identical Dual Xeon E5462 compute nodes connected via a DDR InfiniBand Switch. The timing tests were performed using both NVIDIA Tesla 1060 and Tesla $2050^{13}$ GPUs. Table 1 shows the details of the datasets used to evaluate the framework performance. The tests were performed with different numbers of nodes ranging from 2 to 64 (each with two GPUs), with the data size limiting the minimum number of nodes used. Our test cases include different astronomical data types, including dark matter simulation data [smoothed over a structured grid using the cloud-in-cell technique (Hockney and Eastwood 1988) to show the applicability of our approach to visualize other astronomical data types] and spectral data cubes from different astronomical surveys.

\footnotetext{
13 http://www.nvidia.com/docs/IO/56483/Tesla_C1060_ boardSpec_v03.pdf
} 


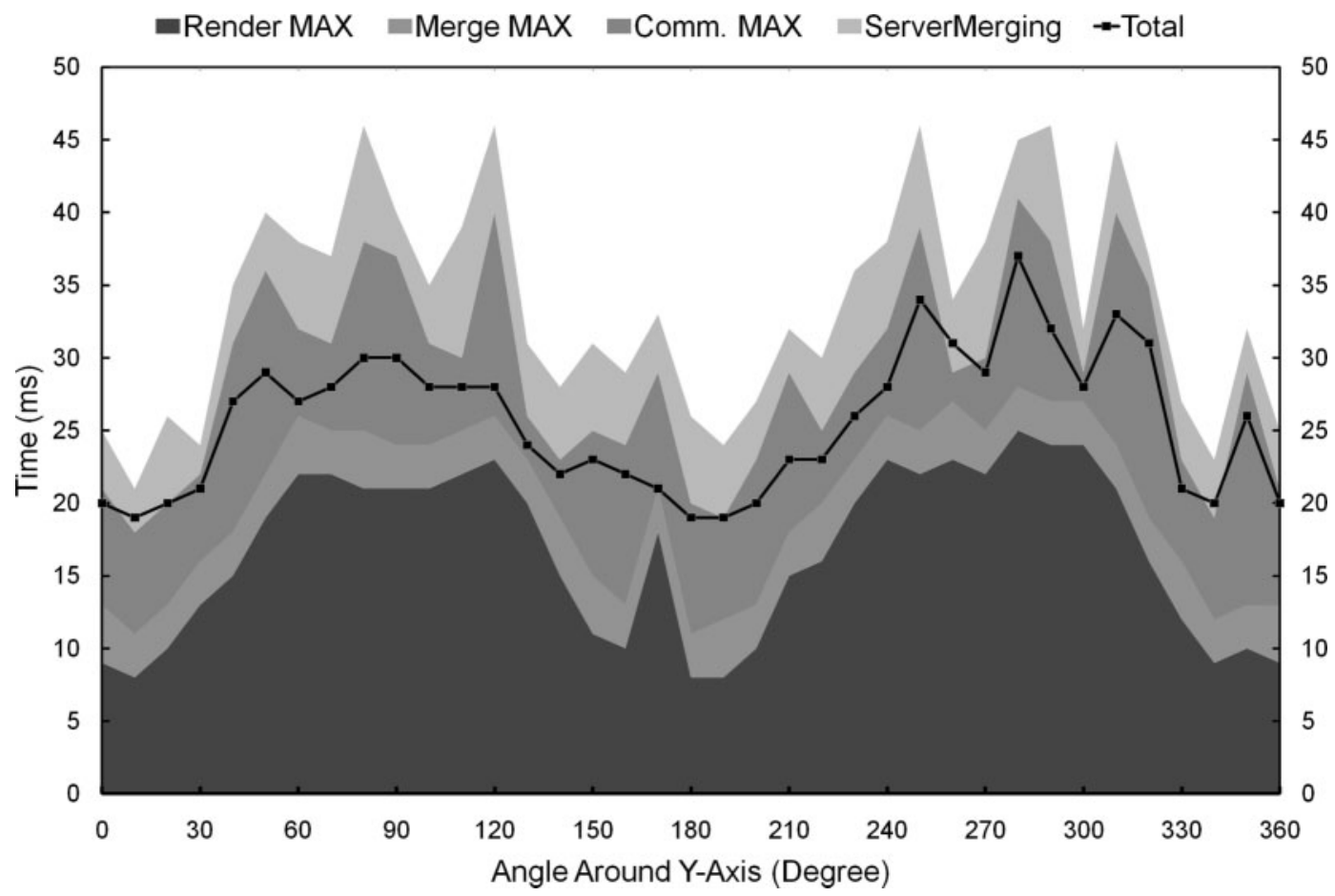

Figure 5 Single frame-rendering time for different cube rotation angles. The timing measurements were done for the Scaled GASS Cube (203.8 GB) on 65 workstations (one acts as a server) and 128 GPUs.

Due to the overlapping between communication and computation and the shared memory-access synchronization, it is hard to find an exact equation to govern the total frame-rendering time in terms of its sub-rendering processes. The otal frame-rendering time (TR) is directly related to the maximum GPU rendering time, the maximum local compositing time, the maximum communication time, and the total server compositing time. Figure 5 shows the relation for the scaled GASS dataset rendered with 64 nodes (128 GPUs), with the measured total framerendering time displayed as a function of cube orientation. The different communication patterns and the overall cluster usage (e.g. from other applications) during our tests may slightly affect the communication delays and hence the final rendering time of each frame. To reduce the impact of such effects, each data point is the median of 10 rendering runs with the same rotation angle. It is clear that the GPU rendering time is the leading factor, varying with the cube orientation (defined in the term of the rotation angle around the $y$-axis). This change in the data-cube orientation affects the size of the rendering rectangles, the amount of overlapping between them, the length of the rays cast, and the number of samples calculated for each of the rays. Due to this variation, we use the $75 \%$ percentile to represent the estimated framerendering time for each data cube.

Figure 6 shows a summary of the timing tests performed using all the test cubes over number of nodes from 2 to 64 , with an output frame size of $1024 \times 1024$, for the Tesla 1060 GPUs. In general, the total frame-rendering time is reduced by the introduction of new GPU nodes; however, the effect of this reduction reaches a critical point where the increase in the GPU nodes slightly increases the total rendering time or else appears to keep it constant. At this critical point, usually the time spent in ray-casting and merging is much lower than the communication overhead, because of the tiny size of the problem (e.g. sub-data cubes occupy less than $1 \%$ of the GPU memory in the case of the 4 GB Nbody simulation cube with 32 nodes).

Figure 7 shows the effect of increasing the output frame size from $1024 \times 1024$ to $2048 \times 2048$ on the total rendering time for different test cubes with 64 nodes and Tesla 1060 as the GPU unit, and shows the same test with a comparison between the timing on Tesla 1060 and 2050. The output frame size is the major factor in determining the number of rays in the ray-casting algorithm, and affects the data size communicated in each frame. The usage of Tesla 2050 instead of Tesla 1060 (with an increase around $180 \%$ in the number of GPU cores but with lower memory size and core frequency) gives a reduction in the total rendering time by $35 \%$ on average (ranging from $10 \%$ to $67 \%$ ).

\subsection{Discussion}

We performed scalability and performance tests for the proposed framework for different test cases, different GPU architectures, and different output resolutions. A frame rate larger than $10 \mathrm{fps}$ is achievable even with an output resolution exceeding the standard $1024 \times 1024$ of a typical desktop monitor. The system scales with an increase in the GPU count until a certain critical point where the problem is too tiny for the GPUs to solve: in 


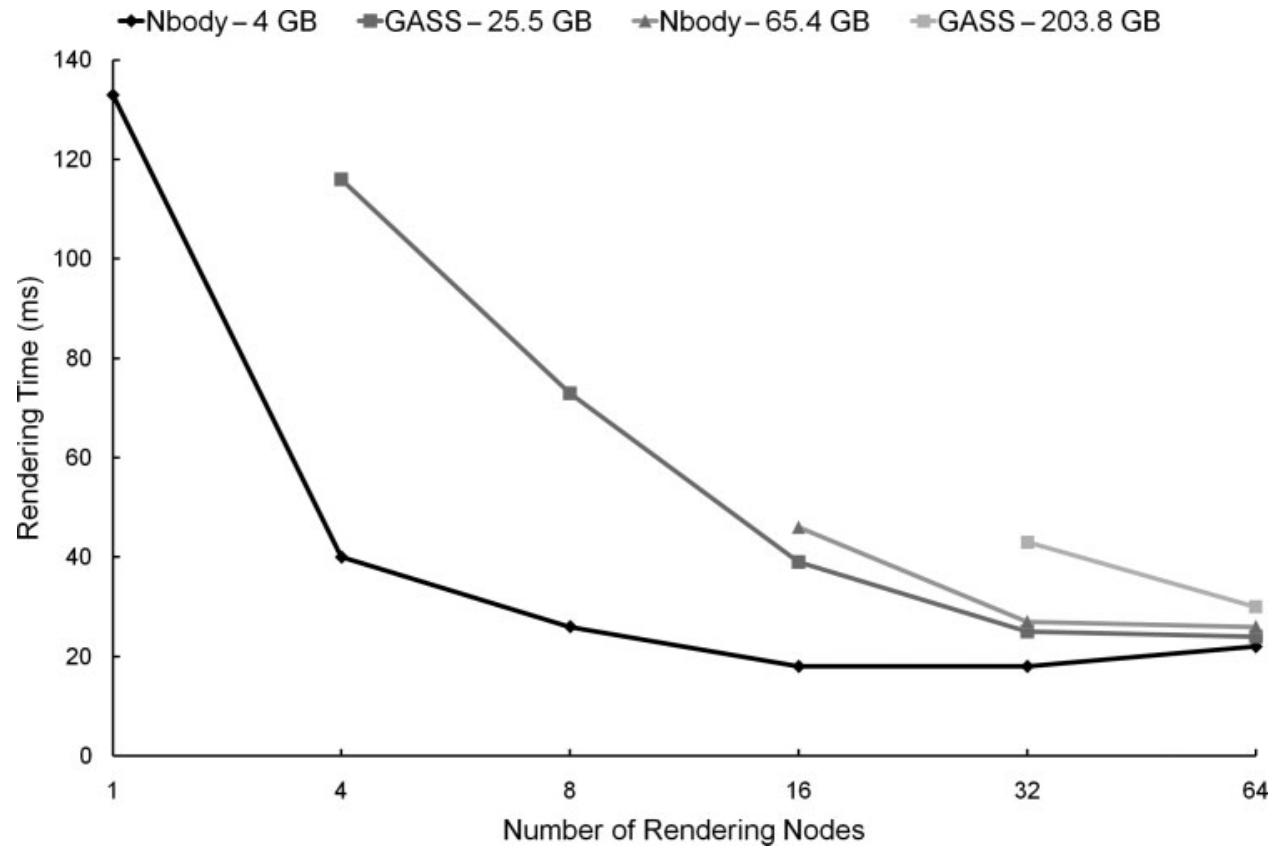

Figure 6 Frame-rendering times for the different test cases with different numbers of rendering nodes, using Tesla 1060 GPUs and an output frame size of $1024 \times 1024$.

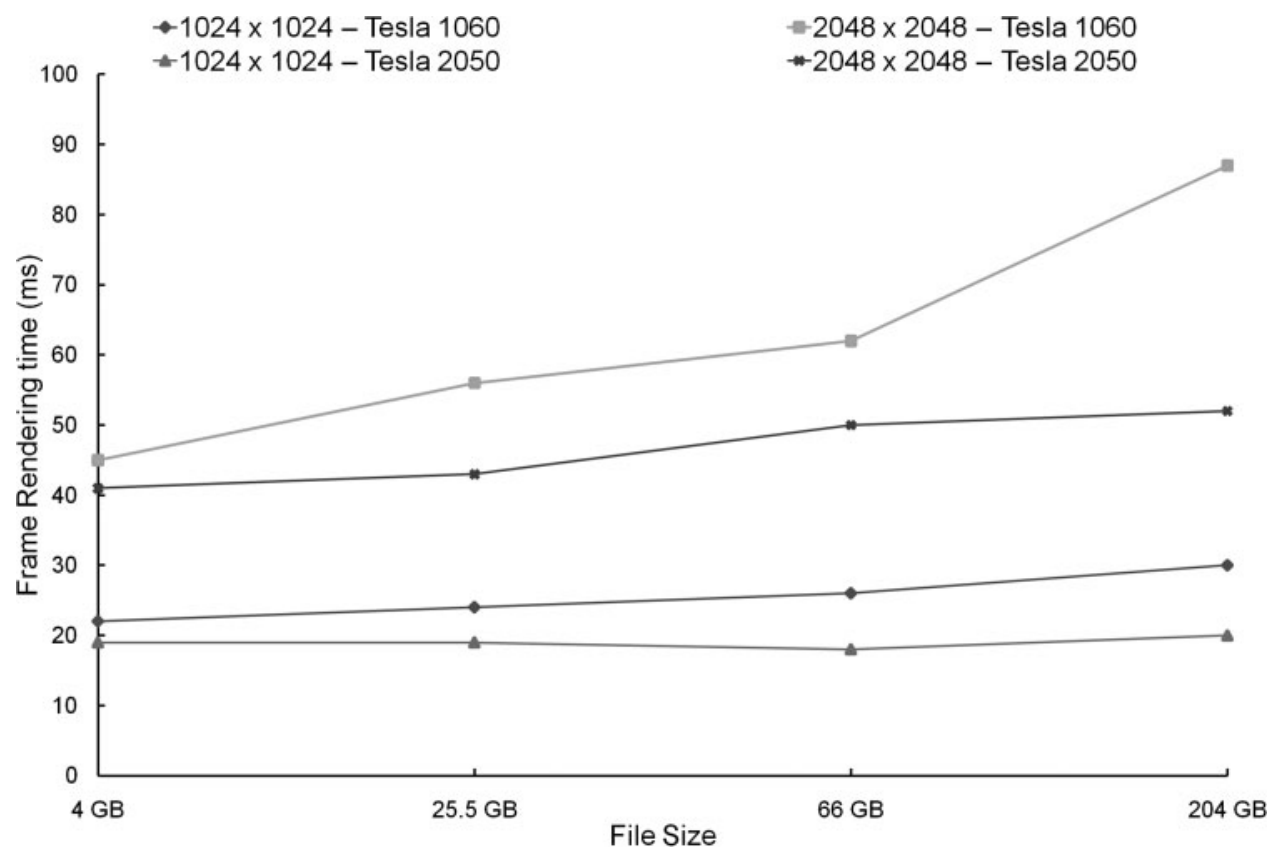

Figure 7 The effect of increasing the output frame resolution on the rendering time from $1024 \times 1024$ to $2048 \times 2048$ for different test cases on 64 rendering nodes with Tesla 1060 and 2050 GPUs.

such cases the communication time exceeds the GPU rendering and compositing time, and may cause a slight increase in the total frame-rendering time. The proposed system enables us to render datasets beyond the current single machine memory limit with a frame rate up to $30 \mathrm{fps}$ for an output size of $1024 \times 1024$ and gives promising results for higher output sizes of $2048 \times 2048$ (despite the current lack of standard desktop displays capable of showing such an image at full resolution).
The main disadvantage of this system is the need for GPU memory that matches the amount of data to be rendered. We think the in-core solution is the best available alternative to achieve a real-time volume rendering and high level of interactivity for terabyte-order datasets. The huge dataset size, especially with the hard disk I/O speed as a limiting factor, is very hard to render using out-of-core methodologies. Also, the use of a multiresolution solution is not practical due to the high 
dynamic range inherent in many astronomical spectral data cubes, the lack of well-defined object boundaries, and the low-signal to noise ratio data values. Furthermore, introducing interactive quantitative visualization along with the qualitative visualization, which is one of our main future work goals, needs the whole dataset to be available in memory to offer a near real-time response for user queries. We expect the memory-limiting condition to be relaxed during the next years with advances in GPU architectures and the increase in available memory per GPU. Most critically, from a financial and cluster management perspective, the number of GPUs needed to render a certain dataset is much fewer than the number of CPU cores needed to render the same dataset.

Using data migration or dynamic load balancing may not be effective in our case, because of the memory limitation that enforces the maximum amount of data that can be stored in the GPU memory. Also, the communication overhead with such data migration may slow down the rendering operation, especially when a highresolution output is required. Static load balancing may be useful to reduce the variation of the frame-rendering time between different orientation angles. The overlapping between communication and computation will reduce the effect of such load balancing. The server has no expectation for a certain node to be faster, and there is no direct relation between that and the final rendering time. A near-cubic data partitioning schema (where the ray lengths and the rendering rectangle areas are almost the same with different cube orientation) minimizes the differences between rendering time for each orientation angle. We leave further static load balancing investigations as a future work.

\subsection{Future Work}

Changing the viewer application into a web-based application using Java3D, FLASH, or HTML5 is one of the steps to enable wider usage of remote visualization in astronomy. Such platform changes are easily integrated with the current framework. Also, enabling quantitative data visualization techniques with more interactivity and control given to the user is an important addition to enable better scientific results and improved knowledge discovery. Additionally, more work is needed to develop a customized astronomical data-transfer function that can suppress the noise and enable discoveries in low signalto-noise ratio values.

\section{Conclusion}

We presented an enhanced framework to volume-render larger-than-memory spectral data cubes. The framework utilizes a hybrid infrastructure of shared- and distributedmemory high-performance computing architectures to enable interactive rendering of datasets that exceed single-machine memory limits. The framework utilizes an optimized version of the ray-casting algorithm and the volume-bricking data-partitioning mechanism to distribute the volume-rendering task over a cluster of GPU-powered workstations connected over a high-speed network. Using available knowledge about the cube orientation and how the data are partitioned over the nodes, the framework optimizes the amount of data that need to be transferred between the different contributing nodes in order to improve the scalability and reduce the framerendering time. Using two-stage compositing reduces the amount of work needed by the server and reduces the total amount of data needed to be transferred to prepare each frame. Using GPUs as the main processing element for the rendering and compositing processes reduces the total rendering time and removes the compositing bottleneck. Moreover, using GPUs reduces the number of nodes needed, the cost needed to visualize such large data cubes, and the communication overhead required.

The framework enables remote visualization by separating the actual results display from the rendering computations. This remote visualization facility can be used to enable a wider usage of the service for geographically distributed users without the need for tedious moving of data between collaborators. Also, this separation enables the usage of different viewing platforms including the Web. Different timing analyses were made to test the framework performance and scalability. The framework was able to render data cubes up to $204 \mathrm{~GB}$ in size at 30 frames per second using 128 GPUs. Our timing tests also investigated the effect of increasing the output resolution and upgrading the GPUs from Tesla 1060 to Tesla 2050. We anticipate, based on these results, that the framework can continue its scalability with a little increase in the communication overhead to render larger data cubes with a comparable performance.

Although our focus has been on volume-rendering spectral data cubes from radio astronomy, we contend that our approach has an applicability to other disciplines where close to real-time volume rendering of terabyteorder 3D data sets is a requirement. For astronomers, the good news is that real-time interactive visualization of terascale spectral data cubes is within reach.

\section{Acknowledgments}

We thank Dr Tim Cornwell (ATNF) and Dr John A Taylor (CSIRO) for providing us with access to the CSIRO GPU cluster. We thank Dr Virginia Kilborn, Dr Emma Ryan-Weber, and Dr Gregory Poole (Swinburne University of Technology), Dr Russell Jurek and Dr Naomi McClure-Griffiths (ATNF-CSIRO), and Dr Tara Murphy (Sydney University) for providing sample data cubes, useful discussions, and suggestions.

\section{References}

Becciani, U., Antonuccio-Delogu, V., Gheller, C., Calori, L., Buonomo, F. \& Imboden, S., 2000, Arxiv preprint (astro-ph/ 0006402)

Becciani, U., Antonuccio-Delogu, V., Buonomo, F. \& Gheller, C., 2001, Arxiv preprint (astroph/0101447) 
Becciani, U., Gheller, C., Antonuccio, V., Ferro, D. \& Melotti, M., 2003, MmSAIS, 1, 80

Beeson, B., Barnes, D. \& Bourke, P., 2003, PASA, 20, 300

Booth, R., De Blok, W., Jonas, J. \& Fanaroff, B., 2009, Arxiv preprint (arXiv:0910.2935)

Comparato, M., Becciani, U., Costa, A., Larsson, B., Garilli, B., Gheller, C. \& Taylor, J., 2007, PASP, 119, 898

Eilemann, S., Makhinya, M. \& Pajarola, R., 2008, in ACM SIGGRAPH ASIA 2008 courses (New York: ACM), 44

Gheller, C., Buonomo, F., Calori, L. \& Becciani, U., 2002, in Proceedings of Joint Eurographics-IEEE TCVG Symposium on Visualization (Barcelona: Eurographics Association), 1

Goel, V. \& Mukherjee, A., 1996, The Visual Computer, 12, 26

Gooch, R., 1995, in Proceedings of the 6th Conference on Visualization '95 (Washington, DC: IEEE Computer Society), 374

Hassan, A. H. \& Fluke, C., 2010, PASA, 122, 119

Hassan, A., Fluke, C. \& Barnes, D., 2011, NewA, 16, 100

Hockney, R. \& Eastwood, J., 1988, Computer Simulation Using Particles (London: Taylor \& Francis)

Humphreys, G., Houston, M., Ng, R., Frank, R., Ahern, S., Kirchner, P. \& Klosowski, J., 2008, in ACM SIGGRAPH ASIA 2008 courses (New York: ACM), 43

Jin, Z., Krokos, M., Rivi, M., Gheller, C., Dolag, K. \& Reinecke, M., 2010, ArXiv 1004.1302

Johnston, S., et al., 2008, ExA, 22, 151

Kaehler, R., Wise, J., Abel, T. \& Hege, H., 2006, in Eurographics IEEE VGTC Workshop on Volume Graphics, ed. R. Machiraju \& T. Moller (Boston: Eurographics Association), 103

Kaufman, A., 1998, SIGGRAPH Course notes, Advances in Volume Visualization 24

Lacroute, P. \& Levoy, M., 1994, in Proc. 21st Annual Conference on Computer Graphics and Interactive Techniques (New York: ACM), 451

Levoy, M., 1988, ICGA, 8, 29

Levoy, M., 1990, ACM Transactions on Graphics (TOG), 9, 245

Lombeyda, S., Moll, L., Shand, M., Breen, D. \& Heirich, A., 2001 , in PVG '01: Proceedings of the IEEE 2001 Symposium on
Parallel and Large-Data Visualization and Graphics (Piscataway: IEEE Press), 115

Magnor, M., Hildebrand, K., Lintu, A. \& Hanson, A., 2005, in IEEE Visualization 2005, VIS 05 (Washington, DC: IEEE Computer Society), 255

Marmitt, G., Friedrich, H. \& Slusallek, P., 2008, in Computer Graphics Forum, Vol. 27 (New York: John Wiley \& Sons), 1687

Maximo, A., Ribeiro, S., Bentes, C., Oliveira, A. \& Farias, R., 2008, in IEEE/EG Int. Symp. Volume and Point-Based Graph., ed. H. C. Hege, D. Laidlaw, R. Pajarola \& O. Staadt (Barcelona: Eurographics Association), 55

McClure-Griffiths, N. M., et al., 2009, ApJS, 181, 398

Molnar, S., Cox, M., Ellsworth, D. \& Fuchs, H., 2008, in ACM SIGGRAPH ASIA 2008 courses (New York: ACM), 35

Muller, C., Strengert, M. \& Ertl, T., 2006, in Proc. EG Symp. Parallel Graphics Vis. (PGV), ed. A. Heirich, B. Raffin \& L. P. dos Santos (Barcelona: Eurographics Association), 59

Muller, C., Strengert, M. \& Ertl, T., 2007, ParC, 33, 406

Nadeau, D., Genetti, J., Napear, S., Pailthorpe, B., Emmart, C., Wesselak, E. \& Davidson, D., 2001, Computer Graphics Forum, 20,27

Oosterloo, T., 1995, PASA, 12, 215

Oosterloo, T., 1996, VA, 40, 571

Payne, H., Jedrzejewski, R. \& Hook, R., 2003, ADASS, 12, 449

Röttgering, H., 2003, NewAR, 47, 405

Scharsach, H., 2005, Proceedings of CESCG, 5, 67

Schwarz, N., 2007, Master's Thesis, University of Illinois

Strengert, M., Klein, T., Botchen, R., Stegmaier, S., Chen, M. \& Ertl, T., 2006, The Visual Computer, 22, 550

Stuart, J. A., Chen, C.-K., Ma, K.-L. \& Owens, J. D., 2010, in HPDC '10: Proceedings of the 19th ACM International Symposium on High Performance Distributed Computing, ed. S. Hariri \& K. Keahey (New York: ACM), 841

Wallis, J., Miller, T., Lerner, C. \& Kleerup, E., 1989, IEEE Transactions on Medical Imaging, 8, 297

Westover, L., 1990, ACM SIGGRAPH Computer Graphics, 24, 376 